\title{
Galactomannan Detection from Piperacillin-Tazobactam Brands Available in the Brazilian Market
}

\author{
Melissa Orzechowski Xavier ${ }^{1}$, Alessandro Comarú Pasqualotto ${ }^{2,3}$, Valério Rodrigues Aquino ${ }^{1,4}$, Teresa Cristina Teixeira \\ Sukiennik ${ }^{2}$ and Luiz Carlos Severo ${ }^{3,5,6}$ \\ ${ }^{1}$ Post-graduation in Pulmonary Sciences, Universidade Federal do Rio Grande do Sul (UFRGS); ${ }^{2}$ Infection Control Department, Santa Casa \\ Complexo Hospitalar, Porto Alegre; ${ }^{3}$ Conselho Nacional de Desenvolvimento e Pesquisa (CNPq), Brazilian government; ${ }^{4}$ Mycology Laboratory, \\ Hospital de Clínicas de Porto Alegre, Porto Alegre; ${ }^{5}$ Mycology Laboratory, Santa Casa Complexo Hospitalar; Porto Alegre; ${ }^{6}$ Internal Medicine \\ Department, UFRGS; Porto Alegre, RS, Brazil
}

\begin{abstract}
Piperacillin-tazobactam is a broad spectrum antimicrobial agent that can cause false-positive results in the commercial Platelia Aspergillus EIA test. So far, no study has been performed in Latin America to evaluate the clinical implication of this finding. Here we studied the potential for galactomannan detection in piperacillintazobactam batches commercialized in the Brazilian market. Five batches from distinct laboratories were tested in duplicate in the Platelia Aspergillus EIA according to the manufacturer's instructions. Only one drug showed crossreaction at a cut-off of 0.5 . Human serum was spiked with this particular drug aiming to mimic achievable piperacillintazobactam concentrations in the serum. Results were all negative for galactomannan detection, even at high drug concentrations. Results from this pilot study suggest that piperacillin-tazobactam might not be a clinically significant cause of false-positive results in the Platelia Aspergillus EIA test in Brazil.
\end{abstract}

Key-Words: Piperacillin-tazobactam, aspergillosis, Platelia Aspergillus, galactomannan.

Galactomannan (GM) detection has gained importance in the diagnosis of invasive aspergillosis (IA) [1,2]. Several monoclonal antibodies have been developed to detect Aspergillus GM molecules, including EB-A2, the actual basis for a sandwich enzyme immunoassay commercial kit (Platelia ${ }^{\mathrm{TM}}$ Aspergillus EIA-Bio-Rad, USA). Although this test has been available in Europe for roughly a decade, it was not before 2003 that the assay was cleared by the Food and Drug Administration (FDA) for use in the United States [3]. In Brazil, approval was obtained by the national regulatory agency (ANVISA, Associação Nacional de Vigilância Sanitária) in late 2007 [4]. Therefore, experience with GM testing in South America is still quite limited.

The ability of GM testing to contribute to the diagnosis of IA is complicated by several factors. In addition to varied sensitivity [2], test performance is hampered by the occurrence of false-positive results, particularly occurring in patients taking antibiotics of fungal origin $[5,6]$. In many cases, a positive GM result can be obtained by testing directly the antibiotic batch. Piperacillin-tazobactam and amoxicillinclavulanate are usually implicated as the main contributors to false-positive reactions [6-8]. Since IA mostly affects patients with prolonged neutropenia (and piperacillin-tazobactam is one the first-line options for empirical treatment of febrile neutropenia) cross-reactions might be of clinical significance. However, there is a controversy about the frequency and importance of piperacillin-tazobactam contamination with GM [9-12], with some authors arguing that we are now in use of a more pure drug [13]. Since no such a data is available for Received on 9 April 2009; revised 21 September 2009.

Address for correspondence: Dr. Alessandro Comarú Pasqualotto. Av Independência 75, Hospital Dom Vicente Scherer, Serviço de Controle de Infecção Hospitalar. Porto Alegre, 90035-150, Brazil. Phone: +55 51 99951614. Fax: +55 51 32148629. E-mail: pasqualotto@santacasa.tche.br.

The Brazilian Journal of Infectious Diseases

2009;13(5):353-355. (C) 2009 by The Brazilian Journal of Infectious Diseases and Contexto Publishing. All rights reserved.
South America, here we investigate the relevance of piperacillin-tazobactam contamination with GM by testing piperacillin-tazobactam batches available in the Brazilian market.

\section{Material and Methods}

A list of companies manufacturing piperacillin-tazobactam in Brazil was initially obtained at the ANVISA website. We were able to get a vial from 5 out of the 7 branches available in the Brazilian market. These were named drugs A, B, C, D, and E.

Lyophilized drugs were diluted as for clinical use in sterile $\mathrm{NaCl} 0.9 \%$ to a final concentration of $45 \mathrm{mg} / \mathrm{mL}$. Platelia Aspergillus test was performed according to manufacturer's instructions. In summary, $300 \mu \mathrm{L}$ of the reconstituted drug was pre-treated as for serum samples. GM testing was performed in duplicate and in two laboratories by distinct scientists who were blinded to the other laboratory results. Sodium chloride was used as a negative control, in addition to the negative control provided in the Platelia Aspergillus kit. GM results were expressed as optical densities (OD) - samples were considered positive when the ratio between the OD observed for the sample and the mean cut-off OD was $\geq 0.5$.

In order to evaluate the potential for false-positive GM results in clinical samples, human serum was spiked with piperacillin-tazobactam using drug samples that showed positive results in the GM testing. Serum used for this experiment was known to be negative for GM (OD of 0.28). Different concentrations of piperacillin-tazobactam were tested, mimicking achievable peak and trough levels after a standard $4.5 \mathrm{~g}$ dose: $600 \mu \mathrm{g} / \mathrm{mL}, 300 \mu \mathrm{g} / \mathrm{mL}, 150 \mu \mathrm{g} / \mathrm{mL}$ and 75 $\mu \mathrm{g} / \mathrm{mL}$ [10]. In this experiment, $300 \mu \mathrm{L}$ of serum was also used as a negative control.

\section{Results}

Results of this study are summarized in Table 1. Apart from drug B, all other drugs tested negative in GM assay. Results were consistent even when tested by different 
Table 1. Optical density indexes of galactomannan as determined by the commercial Platelia Aspergillus EIA in five brands of piperacillin-tazobactam commercialized in Brazil.

\begin{tabular}{|c|c|c|c|c|c|c|c|c|}
\hline \multicolumn{2}{|c|}{ Piperacillin-tazobactam } & \multicolumn{3}{|c|}{ Laboratory 1} & \multicolumn{3}{|c|}{ Laboratory 2} & \multirow{2}{*}{$\begin{array}{c}\text { Final results } \\
\text { Mean }\end{array}$} \\
\hline Drug & Batch & Test 1 & Test 2 & Mean & Test 1 & Test 2 & Mean & \\
\hline A & 133881E & 0.50 & 0.31 & 0.40 & 0.22 & 0.18 & 0.20 & 0.30 \\
\hline B & 006373 & 0.88 & 0.86 & 0.87 & 0.74 & 0.74 & 0.74 & 0.80 \\
\hline C & C09341 & 0.26 & 0.19 & 0.22 & 0.25 & 0.22 & 0.23 & 0.23 \\
\hline D & 7100839 & 0.37 & 0.40 & 0.38 & 0.36 & 0.30 & 0.33 & 0.35 \\
\hline $\mathrm{E}$ & 0760133 & 0.20 & 0.26 & 0.23 & 0.16 & 0.20 & 0.18 & 0.20 \\
\hline
\end{tabular}

laboratories. Drug B was positive to GM detection in all tested wells, with GM OD indexes ranging from 0.74 to 0.88 (mean values of 0.80 ). Sodium chloride used to dilute drugs tested negative.

Spiking human serum with different concentrations of drug $B$ resulted in negative GM results (OD of $<0.5$ ). Mean GM OD indexes for $600 \mu \mathrm{g} / \mathrm{mL}, 300 \mu \mathrm{g} / \mathrm{mL}, 150 \mu \mathrm{g} / \mathrm{mL}$ and 75 $\mu \mathrm{g} / \mathrm{mL}$ of piperacillin-tazobactam were $0.38,0.37,0.37$ and 0.21 , respectively.

\section{Discussion}

To the best of our knowledge this is the first study evaluating cross-reaction in the Platelia Aspergillus EIA test using piperacillin-tazobactam batches commercialized in Brazil. Positive results in the GM test occurred for only one out of the 5 brands tested.

Several studies conducted in other countries have already evaluated the amount of GM contamination in piperacillin-tazobactam vials and the clinical implications for these findings. Results were somehow contradictory, suggesting that variation may occur within brands and even within different batches [6,9,10,12-17]. For instance, Singh et al. [10] tested 3 different batches of piperacillintazobactam from a single manufacturer in Pittsburgh and found very high positive indexes in all samples evaluated (OD of >5.2). Similarly, Aubry et al. [6] detected positive GM indexes in 7 out of 10 batches of piperacillin-tazobactam in France. Median GM index in their study was 2.5, ranging form 0.7-7.8. Also in France, Bart-Debasse et al. [5] described positivity in all piperacillin-tazobactam batches tested $(\mathrm{n}=10)$, with OD index ranging from 0.9-4.3. Similarly, an Italian study [9] found positive GM index in 26/30 (86\%) piperacillin-tazobactam batches evaluated. Some other authors however have questioned these findings, stating that false-positive results are of limited clinical relevance $[12,15]$. In addition, one study tested several piperacillintazobactam batches and found that their GM content was reduced over the time of study. The authors speculated about the possibility of a modification in the manufacturing process to have occurred over time, resulting in more purity and less GM contamination [13].

A study on the kinetics of GM decrease after cessation of $\beta$-lactam antibiotics showed that median time for a negative Platelia Aspergillus EIA test result was 5.5 days. Quite impressively, results may remain falsely positive for up to 30 days [6]. Due to the possibility of cross-reaction with piperacillin-tazobactam in the Platelia Aspergillus EIA test some authors have suggested that testing of suspected antibiotic batches remains the only indicator of possible false EIA positivity [5]. Others have hypothesized that EIA false-positivity might be reduced by sampling serum at trough piperacillin-tazobactam levels or prior to the administration of the next dose $[10,11,13]$. In order to minimize false-positive results in the Platelia Aspergillus GM test in patients on piperacillin-tazobactam, separate tubes of the central venous line should be used for blood sampling and for administration of $\beta$-lactam antibiotics [13].

'Drug B' was the only piperacillin-tazobactam that resulted in a positive reaction in the Platelia Aspergillus EIA in this study. Conversely, at achievable concentrations of piperacillin-tazobactam in serum we did not observe any cross-reaction with this antibiotic. Drug concentrations ranging from $75-600 \mu \mathrm{g} / \mathrm{mL}$ resulted all in GM OD of $<0.5$, which was similar to negative controls.

Some limitations of this study deserve to be mentioned. First of all, only a single batch of each brand of piperacillintazobactam was tested - as already stated, marked variation in GM contamination might occur amongst batches. Secondly, it has been already shown that the kinetics of GM may vary according to the duration of antimicrobial treatment, with GM accumulating after time [5, 8]. Also, the amount of GM in the antibiotic batches does not always correlate with GM levels in vivo [13].

In conclusion, this pilot study suggests that treatment with piperacillin-tazobactam might not be an important cause of false-positive results in the Platelia EIA test in Brazil. More data is clearly needed on this field. Ideally, the amount of GM should be checked in each hospital, using different batches of piperacillin-tazobactam.

\section{Acknowledgements}

This study was supported by a Grant from the Conselho Nacional de Desenvolvimento Científico e Tecnológico (CNPq, Brazilian government). We thank Wyeth, BioChimico, Eurofarma, Novafarma, and Cellofarm for providing the antibiotics batches used in the study. 


\section{References}

1. De Pauw B., Walsh T.J., Donnelly J.P. et al. Revised definitions of invasive fungal disease from the European Organization for Research and Treatment of Cancer/Invasive Fungal Infections Cooperative Group and the National Institute of Allergy and Infectious Diseases Mycoses Study Group (EORTC/MSG) Consensus Group. Clin Infect Dis 2008; 46: 1813-21.

2. Aquino V., Goldani L.Z., Pasqualotto A.C. Update on the contribution of galactomannan for the diagnosis of invasive aspergillosis. Mycopathologia 2007; 163: 191-02.

3. FDA news. FDA Clears Rapid Test for Aspergillus Infection. Available at http://www.fda.gov/bbs/topics/NEWS/2003/ NEW00907.html. Accessed on October $31^{\text {st }} 2008$.

4. ANVISA. Resolução n³.288, de 19 de outubro de 2007. Available at http://www.anvisa.gov.br/legis/suplemento/ 221007_suplemento_1.pdf. Accessed on October 31 2008.

5. Bart-Delabesse E., Basile M., Jijakli A.A. et al. Detection of Aspergillus Galactomannan Antigenemia To Determine Biological and Clinical Implications of Beta-Lactam Treatments. J Clin Microbiol 2005; 43: 5214-20.

6. Aubry A., Porcher R., Bottero J. et al. Occurrence and Kinetics of False-Positive Aspergillus Galactomannan Test Results following Treatment with ß-Lactam Antibiotics in Patients with Hematological Disorders. J Clin Microbiol 2006; 44: 389-94.

7. Alhambra A., Cuétara M.S., Oetiz M.C. et al. False positive galactomannan results in adult hematological patients treated with piperacillin-tazobactam. Rev Iberoam Micol 2007; 24: 106-12.

8. Walsh T.J., Shoham S., Petraitiene R. et al. Detection of Galactomannan Antigenemia in Patients Receiving PiperacillinTazobactam and Correlations between In vitro, In vivo, and Clinical Properties of the Drug-Antigen Interaction. J Clin Microbiol 2004; 42: 4744-8.
9. Machetti M., Furfaro E., Viscoli C. Galactomannan in PiperacillinTazobactam: How Much and to What Extent? Antimicrob Agents Chemother 2005; 49: 3984-5.

10. Singh N., Obman A., Husain S. et al. Reactivity of Platelia Aspergillus Galactomannan Antigen with PiperacillinTazobactam: Clinical Implications Based on Achievable Concentrations in Serum. Antimicrob Agents Chemother 2004; 48: 1989-92.

11. Machetti M., Majabo M.J., Furfaro E. et al. Kinetics of galactomannan in surgical patients receiving perioperative piperacillin/tazobactam prophylaxis. J Antimicrob Chemother 2006; 58: 806-10.

12. Orlopp K., von Lilienfeld-Toal M., Marklein G. et al. False positivity of the Aspergillus galactomannan Platelia ELISA because of piperacillin/tazobactam treatment: does it represent a clinical problem? J Antimicrob Chemother 2008; 62: 1109-12.

13. Penack O., Rempf P., Graf B. et al. False-positive Aspergillus antigen testing due to application of piperacillin/tazobactam is it still an issue? Diag Microbiol Infect Dis 2008; 60: 117-20.

14. Penack O., Schwartz S., Thiel E., Wolfgang Blau I. Lack of evidence that false-positive Aspergillus galactomannan antigen test results are due to treatment with piperacillin-tazobactam. Clin Infect Dis 2004; 39: 1401-2.

15. Ozkalemkas F., Ozcelik T., Ozkocaman V. Treatment with piperacillin-tazobactam and Aspergillus galactomannan test results for patients with hematological malignancies. Eur J Intern Med 2007; 18: 79.

16. Alhambra A., Cuétara M.S., Ortiz M.C. et al. False positive galactomannan results in adult hematological patients treated with piperacillin-tazobactam. Rev Iberoam Micol 2007; 24: 106-12.

17. Viscoli C., Machetti M., Cappellano P. et al. False-positive galactomannan Platelia Aspergillus test results for patients receiving piperacillin-tazobactam. Clin Infect Dis 2004; 38: 913-6. 